\title{
Humanities in Medicine: A Qualitative Study of Graduate and Student Experiences of Completing a Student Selected Component
}

\author{
Diane 0'Doherty*, James A. O’Hare, Sarah Hyde, Deirdre McGrath \\ Graduate Entry Medical School, University of Limerick, Limerick, Ireland \\ Email: *Diane.ODoherty@ul.ie
}

How to cite this paper: O'Doherty, D., O'Hare, J. A., Hyde, S., \& McGrath, D. (2019). Humanities in Medicine: A Qualitative Study of Graduate and Student Experiences of Completing a Student Selected Component. Creative Education, 10, 273-287.

https://doi.org/10.4236/ce.2019.102022

Received: November 21, 2018

Accepted: February 16, 2019

Published: February 19, 2019

Copyright $\odot 2019$ by author(s) and Scientific Research Publishing Inc. This work is licensed under the Creative Commons Attribution International License (CC BY 4.0).

http://creativecommons.org/licenses/by/4.0/

(c) (i) Open Access

\begin{abstract}
Background: As a relatively recent addition to medical education, evidence for the medical humanities is still being gathered. This study aims to explore qualitatively medical students and graduates' perceptions of completing humanities Student Selected Component (SSC) and the potential longitudinal impact of such a module. Methods: A qualitative approach was adopted; one focus group took place with current $3^{\text {rd }}$ year medical students $(n=6)$ and two with medical school graduates $(n=11)$. Thematic analysis was completed on the three focus group sessions. Results: Three main themes were highlighted including 1) evolution, 2) rewards, and 3) difficulties of the humanities project. A number of sub-themes were also reported. Findings highlight the importance of completing the humanities project in regards to promoting self-reflection, development of new skills and impact on clinical practice. Perceived lack of artistic skills, time-management and perfectionism were highlighted as challenging areas. Conclusion: The importance of medical student and graduates' experiences of completing a humanities SSC should be recognised and valued, particularly amongst curriculum developers in medical schools.
\end{abstract}

\section{Keywords}

Humanities, Qualitative Research, Student Selected Component

\section{Background}

In an era where all facets of medical curricula are expected to defend their position in an already busy timetable, the medical humanities have come under pressure to justify its inclusion. As a relatively recent addition to medical educa- 
tion, evidence for the medical humanities is still being gathered. There are diverse views on the definition of medical humanities. The authors of this study feel that it is well encapsulated by the study of what it is to be human, beyond what science alone can tell and is at the core of the Medical Humanities $(\mathrm{MH})$ and can encompass the study of philosophy, theology, literature, theatre, music, history, art, dance and cinema (Dennhardt et al., 2016; Walsh, 2016; Bleakley, 2015; Cole, Carlin, \& Carson, 2015; Macnaughton, 2000). There is also lack of consensus on the ways in which the humanities may enhance aspects of a medical curriculum and its integration into medical education; however there is no singular answer as to the purpose of medical humanities in medical education (Bleakley, 2017; Shapiro, 2012). The authors believe that medical-health humanities employ varying disciplines to gain a deeper understanding of health and illness.

The arts and humanities can help build and foster in medical students core competencies critical to clinical practice such as communication skills, empathy, understanding narrative as well as promoting reflection on and critique of the broader context of medicine in society (Dennhardt et al., 2016; Smajdor, Stockl \& Salter, 2010). They do so by improving verbal and non-verbal communication and by promoting active listening through the study of drama and music (Jones, 2014; Macnaughton, 2000). Students may question their own values and attitudes towards their chosen topic, ultimately ensuring a full-circle reflective process (Bolton, 2005). Reflective practice also promotes reflection on human creativity as well as encouraging the critical analysis of one's own work and of the role of history in the evolution of medicine and how this is applies to keeping up to date with new developments in the field (Macnaughton, 2000). Through exposure to humanities projects students become more self-reflective, reflexive and self-critical, and tend also to review their own vulnerability (Fins, Pohl, \& Doukas, 2013; Shapiro et al., 2009). This process can offer meaningful experiences (Shapiro et al., 2009; Shapiro et al., 2006; Bolton, 2005) and in some cases a shift in attitude (Dennhardt et al., 2016).

Whilst there are opposing views on the fostering of empathy in relation to the medical humanities, extensive literature suggests the importance of building empathy amongst medical students (Mangione et al., 2018; Graham et al., 2016; Marshall \& Bleakley, 2009; Garden, 2007). It has been suggested that empathy may be stunted during students' medical education and that this stunting can then follow through to clerkship and residency (Pedersen, 2010). It has been proposed that the humanities may in fact play a key role in building and developing empathy in medical education (Mangione et al., 2018; Pedersen, 2010; Wershof Schwartz, 2009). By encouraging the development of empathic skills humanities projects may have a long term benefit by encouraging resilience which may in turn help reduce risk of burnout (Mangione et al., 2018; Dennhardt et al., 2016; Rotenstein et al., 2016).

If medical students therefore are to receive a well-rounded medical education with the necessary tools to enable their own self-care and reflection in their fu- 
ture clinical practice, one would foresee a central role for humanities projects in medical curricula. Accrediting bodies such as the General Medical Council in the UK and the American Medical Association in the US both recognise reflection as a "as a requisite skill for the new generation of medical providers and as an important tool for assessing the greater array of competencies now acknowledged in medical education" (General Medical Council, 2018; American Medical Association, 2018; Smajdor, Stockl \& Salter, 2010).

Currently however there is limited literature on the impact of completing a humanities project as a medical student (Jones, Kittendorf, \& Kumagai 2016; Lake, Jackson, \& Harman, 2015; Ousager \& Johannsessen, 2010). Much of the existing literature focuses on student selected, voluntarily completed modules (Ousager \& Johannssen, 2010) which only provides evidence for humanities projects through the experiences of willing participants. This study therefore aims to address medical student and graduate perceptions of completing a compulsory humanities project and its potential impact in the short and long term, using qualitative research methods.

The aim of this study is to:

- Explore the perceptions of students and graduates on completing a humanities SSC and to gain an understanding of its short and long term impact.

\section{Methods}

Student Selected Component (SSC) at Graduate Entry Medical School (GEMS) - Context

Student Selected Components (SSCs), formerly known as Special Studies Modules (SSMs) (Riley, 2009) are popular teaching modalities used by medical schools worldwide and these modules are continuously being appraised (Dennhardt et al., 2016).

The GEMS Humanities SSC is a compulsory 3 week period of study that provides students in their third year with the opportunity to explore one or more aspects of the humanities as it relates to medicine. This SSC lies within the Professional Competencies (PC) module, the aim of which is to foster an appreciation of the behavioural sciences relevant to the practice of medicine and to promote those attitudes that are essential for ethical and professional behaviour. The SSC is worth $50 \%$ of the PC module overall ( 6 credits). This domain is integrated within the $3^{\text {rd }}$ and $4^{\text {th }}$ year clinical teaching of a 4-year, graduate-entry, National Framework of Qualifications (NFQ) Level-8, Bachelor of Medicine Bachelor of Science medical degree programme.

During the course of the three week period students have the opportunity to engage in the humanities and undertake a project through self-directed adult learning. This compulsory project gives students the opportunity to select a topic in medicine to be explored in depth through the humanities or arts. Students at this stage will have had early patient contact and been exposed to a wide range of issues in medicine. Students may produce an original work of art, an original 
piece of music and/or dance, an original film, short stories and/or poetry, or an essay on philosophy, psychology, or history of medicine and have a high degree of choice on what topic or medium they use to complete the project. A core component of the project is a reflective essay which is required to accompany all projects. This reflective essay should outline the rationale for the topic and medium chosen and their own personal experience of completing the humanities project. Students are invited to consent to the exhibition of the reflective essay alongside their project. The project and reflection are graded and count towards the overall mark in the PC module. Students are assessed on relevance, creativity, quality and reflection.

\section{Study Overview}

A qualitative study using focus group discussions was undertaken at the Graduate Entry Medical School, University of Limerick during the course of academic year 2017-18. Focus groups were used to gain an in-depth understanding of the topic. It was hoped that participants' differing viewpoints within the groups would allow further useful information to be attained. Focus groups are often cited as invaluable to health research as they yield a significant amount of qualitative data (Parker \& Tritter, 2006; Morgan, 1997).

\section{Ethics}

The study was approved by the Education and Health Science Faculty Ethics Committee (2017_01_05_EHS).

\section{Data Collection}

A purposive sampling strategy was adopted with an independent gatekeeper sending information sheets to all graduates of the medical school since its establishment in $2007(n=615)$ and to all current third year students who had just completed the humanities project. Purposive sampling is used within qualitative research to identify and selecting participants with a purpose, in relation to key criterion (Ritchie \& Lewis, 2003); in this context all participants have all completed a Humanities SSC project. This was delivered via a student portal and using contact details given by graduates post-graduation. Students and graduates then volunteered to take part in the study (See Figure 1).

As outlined in Figure 1, data was sampled from both a student and graduate population via a recruitment email. No financial or other remuneration was

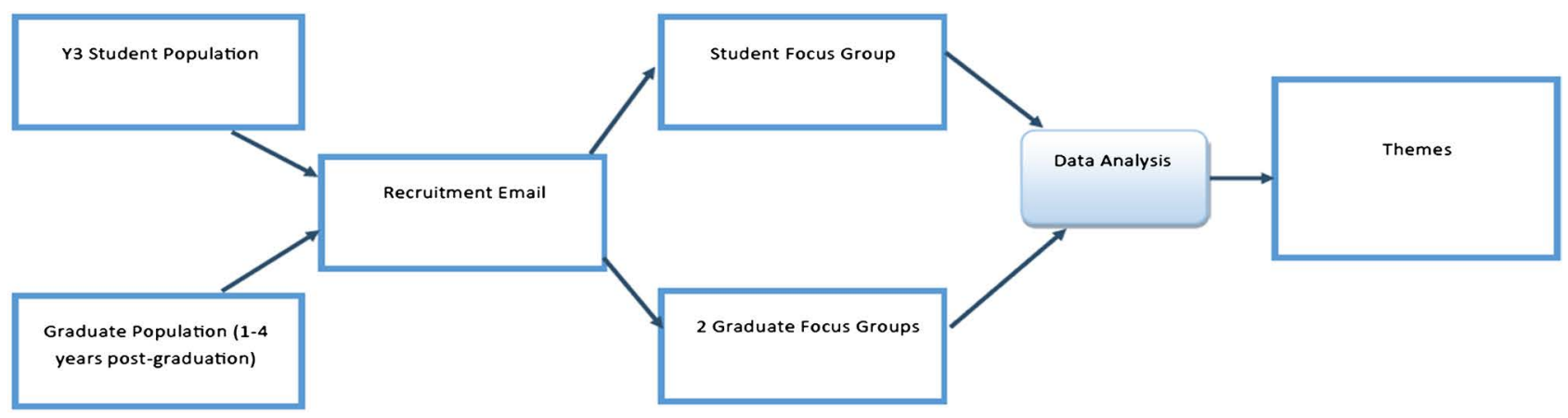

Figure 1. Explanation of data collection process. 
given for participation. All sessions took place between October and November 2017.

A total of three focus group sessions took place after which data saturation was achieved. "Data saturation is reached when there is enough information to replicate the study when the ability to obtain additional new information has been attained, and when further coding is no longer feasible" (Fusch \& Ness, 2015). As outlined in Figure 1, two focus groups were conducted with graduates of the medical school $(\mathrm{n}=11)$, varying in length of time away from the school (1 year [n $=4], 2$ years $[n=3], 5$ years $[n=1], 6$ years $[n=3])$. One focus group was conducted with current third year students who had just completed their SSC project $(\mathrm{n}=6)$. All focus groups took place face-to-face with a group facilitator.

In the context of this study, each focus group discussion was coded and analysed following its completion. Authors agreed that upon reviewing codes (which related to themes outlined in Table 1) of the three focus groups that no new information was being gathered, similar codes were founded and that additional focus group sessions would not warrant any additional information. Some of the initial codes highlighted included personal experience with the topic/medium, skills, time away from medical study and impact of grading.

Focus groups allowed participants to speak freely, disclose any issues they may have had in a non-judgemental environment with a researcher who was experienced in facilitating focus groups but was also an impartial member of staff with no responsibility for assessment of the humanities project. Groups spoke at length about their own positive and negative experiences of completing their SSC and its effects on each individual. Group sizes were also limited to $<8$ participants at a time to allow meaningful discussion to take place. All participants gave their written consent and were made aware that they could exit at any stage and how the data collected would be used for research purposes. Each focus group lasted on average 45 - 90 minutes in total. All focus group sessions were audio recorded and transcribed by a third party company to avoid potential bias in write up. A focus group guide was used to conduct each session (See Appendix 1) that was reviewed by all authors.

\section{Data Analysis}

The data collected was analysed using thematic analysis, according to Braun \& Clarkes' (2006) framework. This process was iterative and inductive, included initial coding of data, revision of these codes by all authors, grouping codes to form themes and discussion of themes. All focus group data was anonymised. The authors reviewed the final codes and themes and a consensus was reached. Findings have been written in accordance with the Standards for Reporting Qualitative Research (SRQR) guidelines (O'Brien et al., 2014). NVivo 11 was used to categorise initial codes and themes.

\section{Results}

Three key themes were created using Braun \& Clarke's (2006) framework as 
Table 1. Overview of core themes and sub themes.

\begin{tabular}{ll}
\hline \multicolumn{1}{c}{ Theme } & \multicolumn{1}{c}{ Sub-themes } \\
\hline 1. Evolution of the Humanities Project & $\begin{array}{l}\text { (a) Personal: Experience and Skills } \\
\text { (b) Practical: Grading \& cost effectiveness }\end{array}$ \\
2. Rewards of the Humanities Project & $\begin{array}{l}\text { (a) Time away from medical study } \\
\text { (b) Develop new interest and relevance to clinical practice } \\
\text { (c) Enjoyment }\end{array}$ \\
3. Difficulties of the Humanities Project & (a) Lack of skills, perfectionism \\
& (b) Strategy-Grading, prioritisation, and competitiveness \\
\hline
\end{tabular}

outlined in Table 1. These three core themes related to the Humanities Project; Evolution, Rewards and Difficulties experienced by participants. Several sub-themes were also highlighted which include the personal and practical experiences, time away from medical study and its relevance to clinical practice and also issues such as lack of creative skills, grading and prioritisation of the humanities project.

1) Evolution of the Humanities Project

a) Personal-Experience and skills

There were two main factors at play when students were considering how to approach their humanities project. Firstly there were the more personal reasons such as previous relevant experiences and also student aptitudes or interests in certain media. Students often reported that their own personal lives and experiences were factors in deciding how best to complete the humanities project.

Graduate 2: "Where I was home in Canada and then could access those things that I liked to do... It was something that's shared with my mum. So it was sort of some quality time then."

Student 5: "I definitely wanted to do an SSC that showcased the mental health of (medical) students because I was kind of thinking that I felt that a lot of maybe staff and faculty and stuff didn't really understand what we were going through..."

Specific skills (or lack of) often lead students to deciding what form their project might take or returning to a former passion or ability which was not currently being utilised in medical school.

Graduate 9: "Lack of artistic talent (laughing) being brutally honest about it!"

Student 6: "I wrote an essay and my undergrad was in English and Psychology so I said, right, my decision was influenced by what I am okay at..."

b) Practical-Grading and cost effectiveness

The other major factors that influenced student decisions were of a more practical nature, such as how they felt that the projects had been marked in the past, and also the practical aspects of creating art, such as cost of materials. Participants spoke extensively about the importance of grading of the humanities project as a factor when deciding on the topic/medium used for their project.

Graduate 6: "I got a bit of advice from the year ahead of me who had scored either the highest or the second in that project. It was after I had already sub- 
mitted my idea so I couldn't change it, and that person said to me 'whatever you do put a positive slant on it. Because that's what I did. And I honestly think that they are graded higher."

Student 3: "It's feeling like, okay, is my idea and this concept good enough to get a good grade or like do I need to try and change it a small bit more to like get a better grade than this because like we're all med students, we' re not like art students."

Participants also perceived that there was a bias towards visual art works by the assessors and that these works were graded higher than written articles or performance pieces.

Graduate 4: "I think by the time I did it, I think I knew that the college, or that the school was putting a little bit of emphasis on something that was kind of impressive to look at... I do think the things that lasted are the things that are most impressive... the sculptures and the art pieces that were most visually impressive."

Graduate 1: "Yeah there is definitely a perception of bias that it's more if you have something tangible at the end. That it's worth more marks."

Many students felt that the cost of such materials and the ability to transport the finished piece were also factors in deciding what medium to use.

Graduate 10: "I also remember buying the stuff for it and thinking this is so unnecessarily expensive when you are a student."

Student 2: "I did a pencil crayon drawing for very similar reasons. I knew I wanted to do it in the summer so it was gonna be art work that I could roll up and bring back because anything that I couldn't roll wouldn't be able to come on the plane with me. I have a bunch of pencil crayons at home because cost again, I didn't want to pay for canvas, I didn't want to pay for paint."

\section{2) Rewards of the Humanities Project}

a) Time away from study to reflect

Students and graduates discussed many of the rewards they felt they gained in completing their humanities project, for example, it offered them time to reflect and gain some insight, something which may not have happened without this opportunity.

Graduate 1: I was focused on... that maybe Pll learn something along the way. So I have to say I did learn, doing it, I got an awful lot of unexpected sort of insight.

This opportunity then allowed some space to reflect and balance what is usually a very hectic study schedule.

Graduate 5: "It's a very intense time and like it just gives you some breathing... some room to breathe and that' s important."

Student 6: "I just think that it's, like maybe doing a project could show people that there's other things than studying that they could be doing to have a better balance."

b) Develop new interests and relevance to clinical practice

Many participants spoke about the benefit of gaining knowledge in new areas while completing their project. For some this creative outlet let them explore 
passions outside of medicine.

Graduate 4: "I was interested in writing, but I never had anything to write about. I never had the time or I never just did it. So I did write a story for my SSC and I enjoyed it. And it gave me an opportunity to do that."

Graduate 3: "I think I got loads out of it because it allowed me to exercise that part of me that I didn't actually get to do in the last three or four years."

Both current students and graduates discussed how completing the SSC affected their clinical work and prepared them for the future. Some participants felt that their approach to patient care benefitted from reflection they had undertaken as part of their project.

Graduate 2: " $P$ d say, in terms of the reflection that I had done it has impacted, in terms of some of my reflection, as a resident, when I was a junior. Particularly on some of the medicine rotation, where I was dealing with certain sick geriatric patients etc."

Student 6: "I think it's such a worthwhile thing to have for students... you see it in like more experienced doctors in the hospital and like in GP, that they have become burned out or they are just tired and just sort of don't enjoy their work as much as they used to so I think it's definitely a healthy outlet to try and maintain your love for medicine as well as like looking after yourself and your own mental health."

A number of students reported that their career choice was influenced by having completed their humanities project on a specific topic.

Graduate 4: “... that gave me a perspective on medical education that 1 ve kept up with. And I still have an interest in... so I benefitted from new perspectives on medical education, more longitudinal and that (have) stayed with me."

c) Enjoyment

Participants spoke about how much enjoyment they felt in doing their SSC project; for some it allowed them to spend time with family, for others it was the process of doing a creative piece from start to finish.

Graduate 2: "I remember just really enjoying, it was time spent with my Mum, who gave me a little bit of help. Mind you it was, it got a little stressful when it's down to like that, the little detail work. But it was enjoyable, in terms of the experience, spending that time."

Student 4: "The process is more important than the end result."

3) Difficulties of the Humanities Project

a) Lack of skills and perfectionism

A commonly discussed issue which participants felt affected them in completing their humanities project is their perceived lack of artistic skills and viewing the project as an "art project".

Graduate 9: "My perception was it was going to be an art project which wouldn't have been my forte."

Graduate 11: "I never want to be an artist I don't want to be, I don't think I need to be."

Student 4: "I m not artistic at all and so I was really concerned about how, 
how it would be marked."

Participants discussed the negative feelings they felt towards their projects, with many identifying lack of control and their own tendencies towards perfection as factors affecting enjoyment of the project.

Student 3: "I just felt like it was never good enough, like I finished and I was like, okay is there any way I can add to this to make it look better... In my eyes I did my best but I still don't like how it looked so I just never felt fully satisfied with it, which to me is like really hard to take."

Student 4: "Your perfectionist tendencies, especially being in medicine you want always like the best... so to like have something that you feel isn't fully representative of your abilities... you' re not totally happy with it. That's not nice."

b) Strategy-Grading, prioritisation, and competitiveness

Participants alluded to developing strategies to manage their project, weighing up the potential benefits against how much time they would need to be spend. Factors influencing this included a perception of the grading process and how much effort would need to be expended to do well.

Graduate 1: "I think judging by third year it's usually a very heavy workload. So you' re always kind of balancing something. It's like your core knowledge versus the other things you need to do. And the SSC sometimes, so the view point that it was kind of a little bit woolly... So like it was worth investing it in. But sometimes I think when you've competing interests as well, it's always a challenge."

Graduate 4: "So you' re always taking away from something. There's always that fear, for medical students... But you' re always like, you know I could be studying for this, instead of doing that."

Many participants felt that the SSC project made students competitive. Authenticity of the work was also questioned.

Graduate 1: "So you could see the natural competitive streak in people emerge. And it kind of it got hot and heated, you know... So I definitely saw some of the competitive nature come out."

Student 3: "It's disheartening when you hear of people that have won awards like that or are in the top ranking of Professional Competencies when you know that they got their sister who is a fashion designer or their cousin who is like in, like a graphic designer or something... to do the art project yourself so it 's a little bit ironic when you hear people trying to cheat the system and then they end up coming out on top at the end of the day. So that's frustrating."

One of the issues highlighted by participants was the perceived subjectivity in grading.

Graduate 2: "There was some question about again, how do you assess... It's something that you' re meant to put together, from your own experiences, your own artistic interests etc. Whether that's through words, whether it's using something tangible, something auditory, or stimulatory that way."

Student 5: “... it is so subjective so you' re always working to what they want as 
opposed to kind of reflecting on you and doing what you want."

These factors affected how the students approached their project and impacted negatively on their enjoyment of the experience.

\section{Discussion}

This study outlines the perceptions of graduates and medical students who had completed a humanities project as a core component of their medical degree training. Our findings suggest that students not only gain a deeper understanding of important concepts but that they also acquire reflective skills relevant to future clinical practice, and find reprieve and time for reflection in their busy medical training as a result. However, they can struggle with competitiveness and prioritising responsibilities when undertaking this type of programme.

Lazarus \& Rosslyn (2003) conducted a study of medical students who had completed an optional humanities SSM. Students were required to submit a written article which related to health and illness, and attend a number of seminars. A reflective journal was used to record the content of the project and also to document the emotional responses each student felt while doing the project. This project was perceived as highly positive by students who opted to do the module. Some students found the module "a relief" in the midst of their medical learning. Our study offered similar insights into student perceptions and attitudes with most students reporting the same feeling of relief even though the SSC was compulsory. At a stressful point in their medical education, this humanities project, it seems, offered time for self-reflection and introspection.

Students sometimes perceive that in order to be a good physician, one must remain in control at all times (Lazarus \& Rosslyn, 2003). As argued by Shapiro (2009) "When things are perceived as being out of control, physicians and physicians in training are left with feelings of impotence and shame, and fear that their inadequacy will be uncovered". Interestingly, participants in our study also highlighted feelings of lack of control as part of their experience of their humanities project. Some participants highlighted their perceived lack of artistic skills and desire for perfectionism as challenges when completing the humanities project. Participants discussed at length the feeling that they could have done better projects.

Participants also noted how they felt stressed in completing the SSC project, particularly those who struggled to prioritise their workload commitments. This in turn, affected their enjoyment in completing the project. Ferguson (2002) reviewed 15 reports of stress in medical students and concluded "that self-actualisation, self-awareness and a sense of self-fulfilment seems to be protective traits, whereas perfectionism, Type A personality and anger suppression were associated with increased vulnerability to stress" (Walsh, 2016). One might hypothesize therefore undertaking a module that promotes the development of self-awareness might pose a difficulty for some. In our study students who strive for perfection within their own work acknowledged that, at times, they struggled internally with not being able to produce something to the highest standard of which they may be 
used to. Whilst this is acknowledged by students and graduates, they manage to overcome this through reflection and being self-aware of how it plays into the future skills and insights needed by a physician.

One of the significant issues faced by students and one recommended for further future review is that of grading of humanities project. SSC projects may be written (poem, fiction), visual (visual arts, traditional photography or video) or a performance piece (original dance or music). Both graduates and current students in our study spoke about the perceived view that visual projects were graded preferentially compared to written or performance pieces. This perception unfortunately has arisen out of hearsay and myth between peers. Lake et al. (2015) have emphasized that assessment of the arts should be done with the most appropriate criteria and methods i.e. innovative qualitative methods rather than using more objective criteria. To judge these arts-based/humanities projects with strict objective competency-based outcomes would lessen the value that these projects can add to a student's medical education and personal development (Lake et al., 2015). Assessment in the Graduate Entry Medical School's Humanities project/SSC is undertaken by two medical educators with an appreciation of the work completed, its creativity and importantly, the use of the reflective essay. Although it may be argued that the myths that have developed around the perceived subjectivity of grading of the SSC can also be said for many other medical school assessments, the authors are well aware that where there is doubt amongst students, that myths can proliferate. Greater clarity therefore is needed in relation to the marking criteria.

A further issue which arose as part of the focus group interviews was the concern around authenticity of projects, despite students signing a statement of originality. Some participants spoke about students having friends or family members with expertise (whether in the arts, design etc) that assisted in producing the work for the students' humanities project. Whilst medical schools can try to eliminate such unprofessional practice by asking students for progress updates to be included as part of the humanities project, unfortunately such practices often remain inherent amongst those who compete and strive for the highest grades.

\section{Conclusion}

This qualitative study suggests that a humanities project, in compulsory and self-directed learning format, allows students the opportunity to develop new skills, revisit previous interests and spend time away from conventional medical study. While one might argue that the study has limited generalisability due to the sample being one medical school (which can limit replicability), the authors wish to emphasize that no other medical school offers a comparable module which in effect will prevent any generalisability. Furthermore as the purpose of qualitative research to gain a deeper understanding of the topic at hand generalisability is not necessarily always possible. Self-selection bias may also be apparent in those who volunteered to take part in focus groups as they may be the participants who already have an interest in the medical humanities. Further- 
more through the development of reflective skills it also appears to have a self-reported long-term, and potential impact for future clinical practice. When designing humanities modules therefore, curriculum developers need to consider 1) the usefulness of having a compulsory versus optional project/SSC, 2) the suitability of directed versus self-direct projects for their particular student population and 3) the role and format of assessment for this type of programme.

\section{Notes on Contributors}

- Diane O'Doherty is a research assistant based in the Graduate Entry Medical School at the University of Limerick.

- James A O'Hare MD, FRCPI, FRCPI is Module lead in Professional Competencies year 3 and 4, Adjunct Associate Professor of Medicine at Graduate Entry Medical School, University of Limerick and Consultant Physician.

- Sarah Hyde MB, BCh, BAO, MRCPI, MICGP, M.Ed. is Lecturer in Medical Education at the Graduate Entry Medical School, University of Limerick and General Practitioner.

- Deirdre Mc Grath, MB, BCH, BAO, MD, MMED, FRCP, FRCI, is Professor \& Director of Education at the Graduate Entry Medical School, University of Limerick and Consultant Respiratory Physician.

\section{Authors' Contributions}

DMG, SH \& JOH designed the study. DOD facilitated the focus groups and initial analysis of data. All authors reviewed the paper, data collected and drafted the paper.

\section{Acknowledgements}

Authors would like to thank $3^{\text {rd }}$ year students and alumni of the Graduate Entry Medical School for their invaluable input and insights which made this study possible.

\section{Conflicts of Interest}

The authors report no conflict of interest. The authors alone are responsible for the content and writing of the article.

\section{Ethics Approval}

The study was approved by the Education and Health Science Faculty Ethics Committee (2017_01_05_EHS).

\section{Availability of Data and Materials Statement}

The datasets used and/or analysed during the current study are available from the corresponding author on reasonable request.

\section{Funding}

There was no funding source for this work. 


\section{References}

AMA (2018). American Medical Association. https://www.ama-assn.org/

Bleakley, A. (2015). Medical Humanities and Medical Education: How the Medical Humanities Can Shape Better Doctors. New York: Routledge.

https://doi.org/10.1111/medu.13236

Bleakley, A. (2017). Six Fallacies That Hinder Development of the Medical Humanities in Medical Education. Medical Education, 51, 126-133. https://doi.org/10.1111/medu.13236

Bolton, G. E. (2005). Reflective Practice: Writing and Professional Development. California: Sage Publications.

Braun, V., \& Clarke, V. (2006). Using Thematic Analysis in Psychology. Qualitative Research in Psychology, 3, 77-101. https://doi.org/10.1191/1478088706qp063oa

Cole, T. R., Carlin, N. S., \& Carson, R. A. (2015). Medical Humanities. Cambridge: Cambridge University Press.

Dennhardt, S., Apramian, T., Lingard, L., Torabi, N., \& Arntfield, S. (2016). Rethinking Research in the Medical Humanities: A Scoping Review and Narrative Synthesis of Quantitative Outcome Studies. Medical Education, 50, 285-299. https://doi.org/10.1111/medu.12812

Ferguson, E. (2002). Factors Associated with Success in Medical School: Systematic Review of the Literature. BMJ, 324, 952-957. https://doi.org/10.1136/bmj.324.7343.952

Fins, J. J., Pohl, B., \& Doukas, D. J. (2013). In Praise of the Humanities in Academic Medicine. Cambridge Quarterly of Healthcare Ethics, 22, 355-364. https://doi.org/10.1017/S0963180113000200

Fusch, P. I., \& Ness, L. R. (2015). Are We There Yet? Data Saturation in Qualitative Research. The Qualitative Report, 20, 1408-1416.

Garden, R. (2007). The Problem of Empathy: Medicine and the Humanities. New Literary History, 38, 551-567. https://doi.org/10.1353/nlh.2007.0037

General Medical Council (2018). https://www.gmc-uk.org

Graham, J., Benson, L. M., Swanson, J., Potyk, D., Daratha, K., \& Roberts, K. (2016). Medical Humanities Coursework Is Associated with Greater Measured Empathy in Medical Students. The American Journal of Medicine, 129, 1334-1337. https://doi.org/10.1016/j.amjmed.2016.08.005

Jones, D. S. (2014). What Can the Arts \& Humanities Offer Medicine. Virtual Mentor, 16, 636-641.

Jones, E. K., Kittendorf, A. L., \& Kumagai, A. K. (2016). Creative Art and Medical Student Development: A Qualitative Study. Medical Education, 52.

Lake, J., Jackson, L., \& Hardman, C. (2015). A Fresh Perspective on Medical Education: The Lens of the Arts. Medical Education, 49, 759-772.

https://doi.org/10.1111/medu.12768

Lazarus, P., \& Rosslyn, F. (2003). The Arts in Medicine: Setting up and Evaluating a New Special Study Module at Leicester Warwick Medical School. Medical Education, 37, 553-559. https://doi.org/10.1046/j.1365-2923.2003.01537.x

Macnaughton, J. (2000). The Humanities in Medical Education: Context, Outcomes and Structures. Journal of Medical Ethics, 26, 23-30. https://doi.org/10.1136/mh.26.1.23

Mangione, S., Chakraborti, C., Staltari, G., Harrison, R., Tunkel, A. R., Liou, K. T., \& Kahn, M. J. (2018). Medical Students' Exposure to the Humanities Correlated with Positive Personal Qualities and Reduced Burnout: A Multi-Institutional US Survey. 
Journal of General Internal Medicine, 33, 628-634.

https://doi.org/10.1007/s11606-017-4275-8

Marshall, R., \& Bleakley, A. (2009). The Death of Hector: Pity in Homer, Empathy in Medical Education. Medical Humanities, 35, 7-12. https://doi.org/10.1136/jmh.2008.001081

Morgan, D. L. (1997). Focus Groups as Qualitative Research (2nd ed.). Thousand Oaks, CA: Sage. https://doi.org/10.4135/9781412984287

O’Brien, B. C., Harris, I. B., Beckman, T. J., Reed, D. A., \& Cook, D. A. (2014). Standards for Reporting Qualitative Research: A Synthesis of Recommendations. Academic Medicine, 89, 1245-1251. https://doi.org/10.1097/ACM.0000000000000388

Ousager, J., \& Johannessen, H. (2010). Humanities in Undergraduate Medical Education: A Literature Review. Academic Medicine, 85, 988-998. https://doi.org/10.1097/ACM.0b013e3181dd226b

Parker, A., \& Tritter, J. (2006). Focus Group Method and Methodology: Current Practice and Recent Debate. IJRME, 29, 23-37. https://doi.org/10.1080/01406720500537304

Pedersen, R. (2010). Empathy Development in Medical Education-A Critical Review. Medical Teacher, 32, 593-600. https://doi.org/10.3109/01421590903544702

Riley, S. C. (2009). Student Selected Components (SSCs): AMEE Guide No 46. Medical Teacher, 31, 885-894. https://doi.org/10.3109/01421590903261096

Ritchie, J., \& Lewis, J. (2003). Qualitative Research in Practice: Public Knowledge and Private Lives. London: Sage.

Rotenstein, L. S., Ramos, M. A., Torre, M., Segal, J. B., Peluso, M. J., Guille, C., Sen, S., \& Mata, D. A. (2016). Prevalence of Depression, Depressive Symptoms, and Suicidal Ideation among Medical Students: A Systematic Review and Meta-Analysis. JAMA, 316, 2214-2236. https://doi.org/10.1001/jama.2016.17324

Shapiro, J. (2009). The Inner World of Medical Students: Listening to Their Voices in Poetry. Oxford: Radcliffe Publishing.

Shapiro, J. (2012). Whither (Whether) Medical Humanities? The Future of Humanities and Arts in Medical Education. Journal for Learning through the Arts, 8, 1-26.

https://doi.org/10.21977/D98111796

Shapiro, J., Coulehan, J., Wear, D., \& Montello, M. (2009). Medical Humanities and Their Discontents: Definitions, Critiques, and Implications. Academic Medicine, 84, 292-198. https://doi.org/10.1097/ACM.0b013e3181938bca

Shapiro, J., Kasman, S., \& Shafer, A. (2006). Words and Wards: A Model of Reflective Writing and Its Uses in Medical Education. Journal of Medical Humanities, 27, 231-244. https://doi.org/10.1007/s10912-006-9020-y

Smajdor, A., Stockl, A., \& Salter, C. (2010). The Limits of Empathy: Problems in Medical Education and Practice. Journal of Medical Ethics, 37, 380-383. https://doi.org/10.1136/jme.2010.039628

Walsh, K. (2016). Oxford Textbook of Medical Education (2nd ed.). Oxford: Oxford University Press.

Wershof Schwartz, A., Abramson, J. S., Wojnowich, I., Accordino, R., Ronan, E. J., \& Rifkin, M. R. (2009). Evaluating the Impact of the Humanities in Medical Education. Mount Sinai Journal of Medicine, 76, 372-38. https://doi.org/10.1002/msj.20126 


\section{Abbreviations}

SSC: Student Selected Component

SSM: Special Studies Module

GEMS: Graduate Entry Medical School

PCs: Professional Competencies

NFQ: National Framework of Qualifications

\section{Appendix 1: Focus Group Guide}

- What were your thoughts the SSC before you had started it?

- What influenced your choice of project for the SSC?

- What subject matter did your SSC explore?

- What did you hope to achieve by choosing the project that you chose?

- Looking back, did you achieve those goals by the end of your project?

- What did you like best about the SSC?

- What did you like least about the SSC?

- What were your thoughts on the Humanities SSC once you had completed it?

- Having completed the SSC how do you think it might influence, or has influenced you as a student and doctor?

- Have you revisited the topics you explored in the SSC since you completed the SSC? 